\title{
Hemichorea and dystonia due to frontal lobe meningioma
}

\author{
Abdul Qayyum Rana, Muhammad Saad Yousuf ${ }^{1}$, Muhammad Zainuddin Hashmi², Zakerabibi Mohammed Kachhvi ${ }^{1}$ \\ Parkinson's Clinic of Eastern Toronto, ${ }^{1}$ Department of Biological Sciences, University of Toronto Scarborough Campus, Toronto, ${ }^{2}$ Department \\ of Biological Sciences, McMaster University, Hamilton, Canada
}

\section{ABSTRACT}

Tumors originating from the meninges, also known as meningiomas, have rarely been known to cause parkinsonian symptoms and other movement disorders. Although some cases of AV malformations causing movement disorders have been described in the literature, not much has been reported about meningiomas in this regard. The aim of this case report is to further highlight the importance of brain imaging in patients with movement disorders for even a benign tumor; and also emphasize the need for a careful movement disorder examination because more than one phenomenology of movement disorders may result from the mechanical pressure caused by a tumor. We present a case report of a patient with a heavily calcified right frontal lobe meningioma. Our patient had irregular, involuntary, brief, fleeting and unpredictable movements of her left upper and lower extremities, consistent with chorea. The patient also had abnormal dystonic posturing of her left arm while walking. This case report highlights the importance of brain imaging as well as careful neurological examinations of patients with benign meningiomas. Moreover, it illustrates the remarkable specificity yet clinical diversity of meningiomas in presentation through movement disorders.

Key words: Dystonia, frontal lobe, hemichorea, meningioma

\section{Introduction}

Meningiomas, first described by Harvey Cushing in 1922, are used to designate tumors originating from the meninges. ${ }^{[1]}$ Epidemiological studies have noticed that meningiomas represent $15 \%$ of all symptomatic and $33 \%$ of all incidental intracranial neoplasms. ${ }^{[2]}$ Furthermore, it has been shown to occur two to three times more commonly in middle-aged females as compared to males. ${ }^{[2]}$ Meningiomas have been known to cause parkinsonian symptoms and other movement disorders, however, this is rare ${ }^{[3]}$ The focus of this case report is on the movement disorders known as chorea and dystonia.

Chorea, derived from the Greek word "dance", is an irregular, rapid, involuntary jerky movement that flows randomly to any part of the body. ${ }^{[4]}$ Chorea can be

\begin{tabular}{|l|l|}
\hline \multicolumn{2}{|c|}{ Access this article online } \\
\hline Quick Response Code: & Website: \\
\hline & www.ruralneuropractice.com \\
\cline { 2 - 2 } & \\
\hline
\end{tabular}

attributed to multiple causes, including Huntington's disease, vascular disorders, electrolyte imbalance, medication infection and autoimmune diseases. ${ }^{[4]}$ When chorea is limited to one side of the body, it is referred to as hemichorea. Dystonia is a disorder characterized by sustained or recurring involuntary muscle contractions generally associated with twisting or repetitive movements and abnormal postures. ${ }^{[4]}$ It can be classified according to age of onset, distribution (focal, multifocal, segmental or generalized), or etiology. ${ }^{[4]}$

Here, we report a rare case of a patient diagnosed with a combination of hemichorea and dystonia on the left side, and a large frontal lobe meningioma on the right side of the brain. It should be noted that although hemichoreas mostly result from a vascular malformation, tumors or tuberculoma in the basal ganglia, hemorrhagic or ischemic stroke, metastasis, non-ketotic hyperglycemia, and hypoglycemia, our patient did not have anything except for a frontal lobe meningioma. ${ }^{[3,5]}$

\section{Case Report}

Our case was a 65-year-old right handed female who started having abnormal limb movements several years ago

Address for correspondence: Dr. Abdul Qayyum Rana, 111-1371 Neilson Road, Toronto, Ontario, Canada -M1B 4Z8.

E-mail: ranaaq@yahoo.com 
for which she was referred to our Movement Disorders Center. The patient had a history of generalized seizures with intermittent loss of consciousness since the age of 44 lasting several minutes.

The abnormal movements involved her left hand and left foot which would occur intermittently when she was awake and disappear when she was asleep. On examination the patient had irregular, involuntary, brief, fleeting, and unpredictable movements of her left upper and lower extremities (hand, fingers and foot) consistent with chorea (see supplementary video). Examination of her gait revealed that she would hold her left arm in dystonic posturing while walking. The patient would smile and laugh at times without any reason due to frontal disinhibition. The rest of her neurological examination including cognitive functioning was unremarkable. The patient's family history was negative for any neurological conditions including Huntington's disease, chorea and other movement disorders. The patient's physical examination showed that she had clinical features of two movement disorders, in particular chorea and dystonia.

The diagnosis of neurological diseases and movement disorders are based on good clinical history and examination. The patient in our case started having abnormal limb movements several years ago, though she was not able to recall the exact starting point. There were no other complains. In terms of the evolution of symptoms there was a very negligible progression of the symptoms of chorea and dystonia in her left hand over the years along with the size of the meningioma.

An MRI of the brain showed a hyperintense irregular lobulated mass of $3.7 \times 3.5 \times 3.2 \mathrm{~cm}$ in the right frontal lobe with minimal leftward midline shift involving the inferior aspect of the left frontal lobe with inhomogeneous enhancement [Figures 1 and 2]. This was consistent with a heavily calcified frontal lobe meningioma. There was no edema or mass effect in the surrounding brain. Her EEG was normal.

Extensive serological investigations for causes of chorea and dystonia were normal. Furthermore, CBC electrolytes, ESR, ANA, rheumatoid factor, thyroid profile, and antiphospholipid antibodies were normal.

\section{Discussion}

Movement disorders in general are uncommon presenting features of brain tumors. ${ }^{[3]}$ Benincasa et al. in

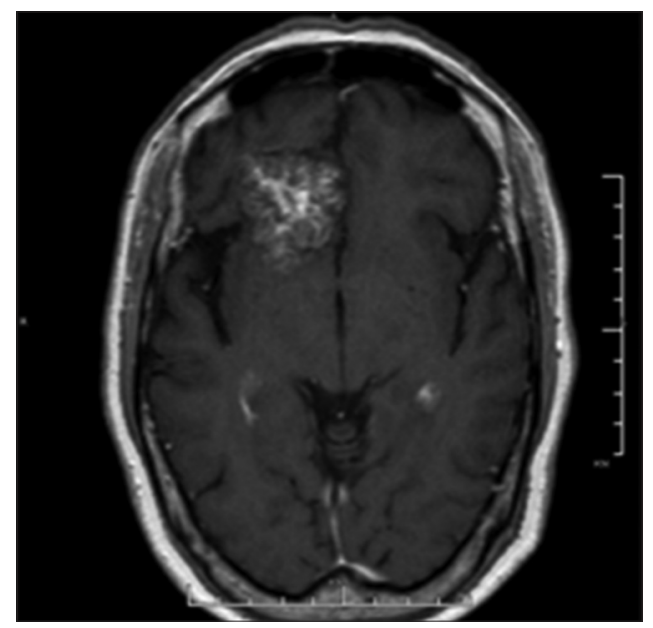

Figure 1: T1-weighted MRI with contrast, axial view demonstrating right frontal lobe lesion with inhomogeneous enhancement

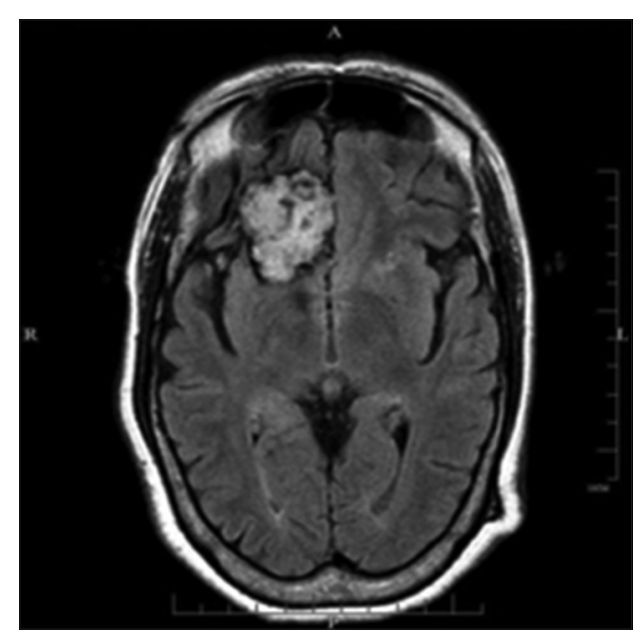

Figure 2: Axial view of FLAIR MRI demonstrating hyperintense right frontal lobe lesion measuring $3.7 \times 3.5 \times 3.2 \mathrm{~cm}$ with minimal mass effect and no edema

2008 presented a case with the right hemiparkinsonism due to a frontal meningioma ${ }^{[6]}$ To our knowledge, it is a very rare phenomenon for a patient with a large frontal lobe meningioma to present simultaneously with hemichorea and dystonia without any other explainable cause. Such a specific case has not yet been cited in the literature. It has been reported that intrinsic basal ganglia and thalamic lesions as well as extrinsic compressive lesions can cause movement disorders, although very rarely. ${ }^{[3]}$ There are two possible mechanisms known to date. Primarily, movement disorders could be a direct result of basal ganglia and/or nigrostriatal pathway compression by the tumor itself or the resulting edema ${ }^{[6,7]}$ Moreover, the symptoms could also be due to impaired blood flow through vasculature of the basal nuclei. ${ }^{[7]}$

In a case reviewed by Bhatoe, ${ }^{[3]}$ there were decreased dopamine binding sites in the caudate secondary to a 
craniopharyngioma. This disorder led to the patient's parkinsonism. In another case of the same review, ${ }^{[3]}$ the authors report the presentation of meningioma of the anterior third ventricle along with parkinsonism. In congruency, the frontal lobe meningioma exerted mechanical pressure on the basal nuclei resulting in the movement disorder symptoms of chorea and dystonia.

Although hemichorea is a very rare form of a movement disorder, it is mostly associated with vascular malformation, tumors or tuberculoma in the basal ganglia, hemorrhagic or ischemic stroke, metastasis, non-ketotic hyperglycemia, and hypoglycemia. ${ }^{[3,5]}$ Our patient only had a heavily calcified large meningioma, as evident from the MRI, which could have compressed or impaired the extrapyramidal system leading to various movement disorders as noted in the prior research. ${ }^{[7]}$

After consultation and weighing the risks as well as benefits of the surgical removal of the large meningioma in our patient, the neurosurgeon advised against the surgical removal of the tumor. As a limitation to the study, a definitive proof of cause and effect is difficult to establish. The chorea and dystonia were purely unilateral and contralateral to the side of frontal meningioma without any other causative factors. Furthermore, the symptoms were non-progressive, over several years, as well as the size of the meningioma on serial MRIs. The meningioma was also the likely cause of her seizures as there was no history of headaches, focal weakness or sensory symptoms on the left side of her body.

\section{Conclusion}

Meningiomas present themselves usually as benign tumors. Nevertheless, the mechanical pressure caused by this enlargement could lead to various movement disorders, which may even coexist as presented in our case. Thus, a thorough neurological examination of even a benign tumor should not be neglected in cases of movement disorders even when the clinical criteria are fulfilled. This case represents the diversity of movement disorders that can result from a benign meningioma, specifically hemichorea and dystonia.

\section{References}

1. Cushing H. The meningiomas (dural endotheliomas): Their source, and favoured seats of origin. Brain 1922;45:282-316.

2. Buetow MP, Buetow PC, Smirniotopoulos JG. Typical, atypical, and misleading features in meningioma. Radiographics 1991;11:1087-106.

3. Bhatoe HS. Movement disorders caused by brain tumours. Neurol India 1999;47:40-2.

4. Brown RH, Victor M, Ropper AH, Adams RD. Adams and Victor's Principles of Neurology. New York: McGraw-Hill Medical Publishing Division; 2005. p. 1382.

5. Paraskebas GP, Vlachos GS, Vassilopoulou S, Anagnostou E, Spengos K, Zis V. Hypoglycemia-induced hemichorea in a patient with Fahr's syndrome. Neurol Sci 2012;33:1397-9.

6. Benincasa D, Romano A, Mastronardi L, Pellicano C, Bozzao A, PontieriFE. Hemiparkinsonism due to frontal meningioma. Acta Neurol Belg 2008; 108:29-32.

7. Salvati M, Frati A, Ferrari P, Verrelli C, Artizzu S, Letizia C. Parkinsonian syndrome in a patient with a pterional meningioma: Case report and review of the literature. Clin Neurol Neurosurg 2000;102:243-5.

How to cite this article: Rana AQ, Yousuf MS, Hashmi MZ, Kachhvi ZM. Hemichorea and dystonia due to frontal lobe meningioma. J Neurosci Rural Pract 2014;5:290-2.

Source of Support: Nil. Conflict of Interest: None declared. 